Recherches en didactique des langues et des cultures

Les cahiers de l'Acedle

$4 \mid 2007$

Notions en questions : Méthodologies de recherche

\title{
Recherche-développement et didactique des langues
}

Nicolas Guichon

\section{OpenEdition}

Journals

Édition électronique

URL : http://journals.openedition.org/rdlc/4903

DOI : $10.4000 /$ rdlc.4903

ISSN : 1958-5772

Éditeur

ACEDLE

Référence électronique

Nicolas Guichon, "Recherche-développement et didactique des langues », Recherches en didactique des langues et des cultures [En ligne], 4| 2007, mis en ligne le 15 janvier 2019, consulté le 11 septembre 2019. URL : http://journals.openedition.org/rdlc/4903 ; DOI : 10.4000/rdlc.4903

Ce document a été généré automatiquement le 11 septembre 2019

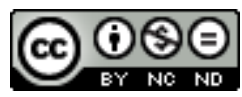

Recherches en didactique des langues et des cultures is licensed under a Creative Commons AttributionNonCommercial-NoDerivatives 4.0 International License 


\title{
Recherche-développement et didactique des langues
}

\author{
Nicolas Guichon
}

\section{Introduction}

1 La recherche sur l'apprentissage médiatisé des langues étrangères place les didacticiens face à deux questionnements majeurs. Le premier concerne la formation de modèles théoriques pour parvenir à des préconisations didactiques (quelles conditions réunir pour développer une compétence langagière ?) tandis que le second s'intéresse à l'évaluation du potentiel de l'offre technico-pédagogique pour l'apprentissage des langues (apprendon bien avec une ressource multimédia?). Les chercheurs soucieux d'étudier ces deux aspects en viennent parfois à recourir à une démarche de recherche-développement qui leur permet de dépasser la seule étape des préconisations pour aboutir à une réalisation qu'ils pourront tester auprès du public cible d'apprenants. Couramment utilisée dans le secteur industriel mais émergente en didactique des langues, cette méthodologie est utilisée pour élaborer et valider des connaissances et les investir dans de nouvelles applications. L'objectif de la présente contribution est d'analyser le potentiel de cette démarche pour la recherche sur l'apprentissage médiatisé des langues.

2 Parce qu'il constitue un point de repère incontournable, l'exemple des concepteurs multimédias du secteur privé permettra, dans une première partie, de délimiter les contours de la recherche-développement et de montrer que leur démarche diverge sensiblement d'une démarche universitaire. Puis, en examinant le type de recherches en didactique des langues qui empruntent cette méthodologie, nous tenterons d'en dégager les traits distinctifs afin de proposer, in fine, une définition de la recherchedéveloppement contextualisée. Enfin, cette contribution se terminera en esquissant quelques propositions pour que le produit des recherches menées en milieu universitaire dépasse le cadre de conception initial en explicitant les enjeux et les difficultés de cette décontextualisation. 


\section{Une méthodologie issue du secteur industriel}

\subsection{Eléments de définition}

3 Comme le note Latour (2005: 410), la recherche-développement est une méthodologie majoritairement utilisée dans l'industrie. C'est particulièrement le cas du secteur industriel producteur de biens et de services à forte valeur ajoutée, c'est-à-dire présentant des innovations ou des améliorations d'ordre technologique.

4 Les entreprises innovantes sont généralement dotées d'un service de recherchedéveloppement qui leur permet de conserver une avance technologique, laquelle peut se révéler déterminante dans certains secteurs d'activité. Si on prend l'exemple du secteur $\mathrm{du}$ jeu vidéo, la recherche-développement est particulièrement intense au début du processus de conception et représente, dans le cas d'un jeu vidéo ambitieux, environ un quart du budget (Le Diberder, 2003). De plus, ce type de produit étant soumis à des cycles très marqués, il exige des améliorations constantes concernant, par exemple, la qualité graphique de l'interface ou l'utilisabilité du système technique sans lesquelles le produit risque de connaître une obsolescence rapide.

5 Le Manuel de Frascati (2002: 56-7) propose une certain nombre de critères d'identification de la recherche-développement dans un service, à savoir le lien avec des laboratoires de recherche, le fait de compter parmi son personnel des doctorants, ou de publier les résultats des travaux. En outre, pour déterminer si un logiciel relève ou non de la recherche-développement, il est spécifié que "son achèvement doit nécessiter un progrès scientifique et/ou technologique et il doit avoir pour objet de dissiper une incertitude scientifique et/ou technologique de façon systématique" ce qui disqualifie, selon les auteurs, "l'utilisation de logiciels pour une application nouvelle ou une finalité nouvelle" (op. cit., p. 53-4).

\subsection{Deux exemples de concepteurs multimédias privés}

6 Dans le secteur du multimédia éducatif français, la recherche-développement telle qu'elle vient d'être strictement définie est assez rare, ce qui s'explique principalement par des raisons économiques. Après une période d'effervescente et de confusion initiale, le secteur de la conception multimédia a connu des changements importants et a vu disparaître beaucoup des premiers acteurs au cours des années 1990 tandis que cette concentration s'accompagnait d'un appauvrissement de la créativité dans les produits proposés (OMPLEP, 2006). Parmi les acteurs privés de ce secteur, deux cas de figure peuvent être retenus car ils se situent aux extrémités d'un continuum sur un axe artisanat-industrie. Il s'agit, d'une part, d'Edulang et, d'autre part, d'Auralog ${ }^{1}$.

7 Edulang est une PME qui compte, en plus de son directeur et d'une équipe d'auteurs qui interviennent ponctuellement, une responsable pédagogique, un infographiste, deux informaticiens et une responsable commerciale. Aucune de ces personnes ne possède de formation universitaire spécifique dans le domaine du multimédia pédagogique. Il existe une collégialité dans le processus de conception. Si on prend l'exemple d'Audioster, dernière production de cette entreprise, la structure pédagogique a été conçue par un universitaire britannique qui remplit le rôle de consultant externe. Le concept de base est ensuite retravaillé par l'équipe d'Edulang selon des critères de faisabilité (économique, informatique, juridique) et d'utilité (pédagogique). Vient ensuite une phase de 
développement d'environ vingt-quatre mois qui débouche sur la mise sur le marché d'une première version, laquelle est amendée grâce aux retours des utilisateurs. La partie développement représente entre 15 et $20 \%$ du chiffre d'affaires et est affectée principalement aux coûts salariaux et à la rémunération des auteurs. L'appellation de recherche-développement n'est, à juste titre, pas revendiquée, le souci d'Edulang étant prioritairement de proposer des ressources pédagogiques pour des enseignants qui n'ont pas toujours les moyens de réaliser un travail de cette envergure.

Auralog, fondée en 1987, est une entreprise qui compte 200 employés et qui s'est très rapidement positionnée sur le marché du multimédia éducatif avec son produit phare, Tell Me More. Ce dernier est basé sur une adaptation du principe de la reconnaissance vocale pour l'apprentissage des langues. La recherche-développement est intervenue dans les premières années du produit (on peut parler à ce moment-là d'une véritable innovation), et a connu depuis des versions différentes avec, en particulier, des réglages pour la qualité de la reconnaissance vocale. La partie développement occupe environ le tiers des employés parmi lesquels on peut dégager trois profils, l'un technique, l'autre pédagogique et le troisième linguistique, mais il n'y a pas de doctorants ni de spécialistes de l'apprentissage au sein de l'équipe. La recherche-développement est clairement identifiée comme un élément stratégique dans le modèle économique et le chiffre d'affaire généré permet de financer des investissements importants dans ce secteur afin d'améliorer l'offre technico-pédagogique. Le positionnement d'Auralog comme un des leaders sur le marché du multimédia éducatif est facilité par un marketing performant et la qualité de l'interface de ses produits.

\subsection{Synthèse}

Ces deux exemples sont représentatifs de la façon dont l'industrie du multimédia éducatif est structurée; d'une part, on trouve une conception de ressources pédagogiques quasi artisanale qui n'est pas sans rappeler la démarche de l'enseignant passionné de pédagogie et d'informatique qui va concevoir seul, ou avec une équipe réduite, et avec des moyens limités un logiciel "sur mesure" destiné à un public particulier. De l'autre, des entreprises ou des maisons d'édition disposant de moyens conséquents pour mettre au point des outils à la finition impeccable destiné à un large public. Quand l'une assume sa proximité avec l'Education Nationale et recherche le label "Reconnu d'Intérêt Pédagogique" délivré par celle-ci, l'autre joue surtout de sa puissance de feu et oriente sa stratégie vers le secteur plus rentable des particuliers et des entreprises sans négliger cependant les clients universitaires.

La conception de ressources pédagogiques sur support numérique se confond donc rarement avec la recherche-développement stricto sensu. Une recherche-développement s'astreint à des exigences scientifiques et méthodologiques et suppose la mise en place d'expérimentations pour valider les choix opérés. Dans le cas de la conception d'une ressource multimédia, les exigences économiques obligent les concepteurs à concevoir un produit destiné à un public aussi large que possible, ce qui conduit à une imprécision dans la définition des besoins et, partant, à des propositions pédagogiques parfois incongrues en termes de moyens offerts pour développer telle ou telle compétence. D'autre part, les évaluations auprès $\mathrm{du}$ public cible se résument souvent à un questionnaire de satisfaction, qui devient ensuite, quand il est favorable, un argument de vente. 
11 Ainsi, les concepteurs multimédias du secteur privé recourent principalement à la recherche-développement pour garder une avance stratégique sur leurs concurrents, mais, d'une version à une autre, ce sont essentiellement des considérations graphiques et ergonomiques qui sont privilégiées. Les aspects didactiques sont la plupart du temps absents de leurs préoccupations et on note même souvent des retours à des visions dépassées de l'apprentissage dans les produits proposés qui font la part belle à des batteries de questionnaires à choix multiple dont le potentiel limité pour l'apprentissage est habilement dissimulé sous des atours modernes.

\section{Recherche-développement et didactique des langues}

12 Les objectifs et les moyens de la recherche-développement diffèrent entre l'industrie et les universités de sciences humaines et sociales. Pour ces dernières, le mode de production des connaissances s'appuie traditionnellement sur la recherche fondamentale, plus rarement sur la recherche appliquée, mais débouche exceptionnellement sur un développement. Toutefois, en raison de son positionnement original, la didactique des langues construit son cadre théorique en recourant à différentes disciplines constituées (linguistique, psychologie, psycholinguistique, sociologie, sociolinguistique, sciences de l'éducation) qu'elle confronte à des pratiques pour produire des connaissances sur l'enseignement/apprentissage de la L2 (Van Lier, 1996: 219). Ainsi, il n'est pas rare qu'une recherche en didactique des langues se construise parallèlement à la mise en place d'une innovation ou à la conception d'un dispositif. C'est ce dernier cas que nous allons maintenant étudier à travers l'analyse de quelques thèses en didactique qui proposent une réflexion sur l'apprentissage des langues et s'accompagnement du développement d'une ressource numérique ou d'un dispositif d'apprentissage médiatisé.

\subsection{Quelques exemples de recherche-développement}

13 Le tableau suivant présente une liste non exhaustive de quatre thèses classées par leurs auteurs dans la catégorie de la recherche-développement et qui, à l'exception de Listening to Sophie, ont abouti à la production d'un logiciel réellement utilisé par des apprenants ${ }^{2}$.

Tableau 1 : Quatre exemples de recherche-développement.

\begin{tabular}{|l|l|l|}
\hline Auteurs & Projet & Production \\
\hline $\begin{array}{l}\text { Mangenot } \\
(1995)\end{array}$ & $\begin{array}{l}\text { Conception d'un "environnement d'écriture" pour faciliter la } \\
\text { production écrite et rendre plus visibles certains processus. }\end{array}$ & $\begin{array}{l}\text { Word Prof (Gammes } \\
\text { d'écriture) }\end{array}$ \\
\hline $\begin{array}{l}\text { Poussard } \\
(2000)\end{array}$ & $\begin{array}{l}\text { Conception d'un produit visant l'apprentissage ou le } \\
\text { perfectionnement de la compréhension de l'anglais oral }\end{array}$ & Listening to Sophie \\
\hline $\begin{array}{l}\text { Rézeau } \\
(2001)\end{array}$ & $\begin{array}{l}\text { Développement d'un didacticiel pour améliorer } \\
\text { l'apprentissage de l'anglais par des étudiants d'histoire de } \\
\text { l'art peu motivés. }\end{array}$ & $\begin{array}{l}\text { English for Art } \\
\text { History }\end{array}$ \\
\hline
\end{tabular}




\begin{tabular}{|l|l|l|}
\hline $\begin{array}{l}\text { Guichon } \\
(2004)\end{array}$ & $\begin{array}{l}\text { Conception d'un dispositif d'apprentissage médiatisé } \\
\text { permettant de développer la compréhension de l'oral }\end{array}$ & Virtual Cabinet \\
\hline
\end{tabular}

14 A travers ces quelques exemples, il est possible de dégager trois particularités. Tout d'abord, ce type de recherche pose plus ou moins explicitement la question suivante: quel dispositif technico-pédagogique est-il possible de concevoir pour faciliter le développement de compétences langagières en L2 ? Les aspects didactiques privilégient des réflexions sur les aides à apporter pour guider l'apprentissage ou sur l'adéquation d'un scénario à un public ou à un objectif tandis que les aspects technologiques favorisent, par exemple, des questionnements sur la qualité des systèmes de gestion des réponses. Ces priorités ne sont pas forcément antinomiques et font même souvent l'objet d'assemblages originaux, mais ce sont toutefois les préoccupations didactiques qui ont l'ascendant sur les aspects technologiques dans toutes ces recherches (cf. Depover et al., 2000).

15 La seconde particularité est de poser l'objet conçu comme une façon de "mettre à l'épreuve " la théorie (Poussard, 2000) voire de valider une hypothèse (Rézeau, 2001, Guichon, 2004). Quand ils ne restent pas à l'état de prototypes, la ressource ou le dispositif conçus ne sont jamais présentés comme la finalité de la recherche. En revanche, tous les chercheurs insistent sur le processus de conception, ce qui permet bien de parler de développement.

16 Enfin, et c'est la dernière particularité, les chercheurs impliqués dans ce type de recherche bénéficient d'une assise institutionnelle ${ }^{3}$ quand ils entament leur recherchedéveloppement et ils possèdent des compétences acquises dans des projets antérieurs ou disposent du concours d'informaticiens. Ceci rappelle qu'une recherche-développement nécessite des compétences spécifiques (technologiques ou humaines quand il s'agit de coordonner une équipe), du temps et des moyens (financiers, humains, matériels, ...) difficiles à trouver sans soutien institutionnel. Ces contraintes pourraient en partie expliquer pourquoi peu de recherches-développements ont été menées depuis dix ans. D'autre part, l'implication du chercheur dans son contexte soulève des questions épistémologiques propres à la didactique. Celle-ci, en effet, est confrontée à des exigences d'utilité sociale, ce qui conduit fréquemment les chercheurs impliqués dans une recherche-développement non seulement à concevoir des ressources ou un dispositif mais aussi à les intégrer dans le contexte institutionnel dans lequel ils évoluent.

17 Les laboratoires transdisciplinaires ${ }^{4}$, comme le Laboratoire de Recherche sur le Langage (LRL) de l'Université de Clermont qui a travaillé à l'élaboration du cédérom Camille, semblent constituer la configuration la plus prometteuse pour ce qui concerne la recherche-développement dans le domaine de l'apprentissage des langues car peuvent alors se croiser des approches et des méthodologies appartenant à chaque discipline qui sont susceptibles de créer des synergies et de déboucher sur des propositions technicopédagogiques ambitieuses.

\subsection{Recherche-action ou recherche-développement}

Quand il s'agit de définir le type de recherche entreprise, on peut remarquer des positionnements plus ou moins affirmés parmi les chercheurs du domaine. Ainsi, Poussard (2000: 7) revendique le terme de recherche-développement pour qualifier son 
travail de recherche. Celle-ci part de l'analyse d'un problème initial et pose les jalons pour développer un logiciel, Listening to Sophie, en puisant des connaissances provenant de différents champs théoriques pour "avancer des propositions et arriver à une production [...] envisagée, a priori, comme un prototype plus qu'un produit fini".

En revanche, pour Rézeau (2001: 7), la recherche-développement est "une variante de la recherche-action" et il annonce, dès l'introduction de sa thèse, son intention de "mener en parallèle une réflexion théorique et le développement d'instruments innovants", une recherche qui, ajoute-t-il, "va jusqu'au bout de la recherche-action, jusqu'au développement d'un instrument utilisable et réellement utilisé sur le terrain". Ainsi, selon les propres termes de Rézeau, la recherche-développement débouchant sur la conception du didacticiel English for Art History est non seulement le prolongement d'une recherche-action, mais elle répond aussi à des demandes d'utilité pédagogique. Il est d'ailleurs notable que Rézeau n'emploie le vocable recherche-développement que deux fois dans sa thèse, alors qu'il fait référence à la recherche-action à de nombreuses reprises. Comme le remarque Mangenot (2002: 128), "la ligne de partage entre recherche théorique, recherche-développement et recherche-action est souvent ténue et les allers-retours d'un cadre théorique ou méthodologique à un autre sont de mise dans la littérature du domaine".

Cette indétermination peut être expliquée par des éléments sociologiques et épistémologiques. La recherche sur les TICE n'a réellement commencé qu'il y a environ trois décennies et $n$ 'a gagné sa reconnaissance universitaire ${ }^{5}$ que depuis une quinzaine d'années avec l'apparition de publications et de colloques propres au domaine. De plus, la recherche sur les TICE se structure autour de deux disciplines (didactique et informatique) dont l'hybridation demeure un objet de méfiance au sein de l'université. On peut lire à ce sujet le seul document de synthèse pour une habilitation à diriger des recherches (HDR) produit à ce jour qui associe recherche-développement et didactique des langues et dont l'auteur admet qu'elle contient des "facettes qui pourront paraître inhabituelles à certains, du moins dans le champ de l'anglistique" (Cazade, $2000: 4$ ).

21 Concernant le statut épistémologique de la recherche-développement, relativement peu de thèses adoptant cette méthodologie ont été écrites et la modélisation de ce type de recherche reste à définir (cf Rézeau, $2001: 3$ ). Contrairement à la recherche-action dont le cadre théorique s'affermit progressivement, la recherche-développement en didactique des langues souffre d'une absence de paradigme par rapport à quoi elle pourrait se définir.

Toutefois, la proximité entre les deux types de recherche est grande. Pothier (2003: 40) montre la complémentarité entre recherche-action et recherche-développement tant que cette dernière est "bien comprise", c'est-à-dire si elle se réfère explicitement aux théories d'apprentissage, et si elle inclut une évaluation méthodique en termes d'apprentissage et, le cas échéant, en termes d'enseignement quand le logiciel développé s'inscrit dans un offre de formation.

Quand la recherche-action cherche à "améliorer les pratiques [d'apprentissage, d'enseignement ou d'accompagnement] grâce à des expériences éclairées et nourries de savoirs théoriques" (Catroux, 2002 : 9), la recherche-développement entreprend des travaux de création "de façon systématique en vue d'accroître la somme des connaissances (...) ainsi que l'utilisation de cette somme de connaissances pour de nouvelles applications" (Manuel de Frascati, $2002: 34$ ). Il est dès lors possible de postuler que, si recherche-action et recherche-développement partagent un objectif sensiblement similaire, la principale différence entre les deux 
méthodologies vient de l'intervention de la technologie comme un élément central qui s'intercale entre le chercheur, l'apprentissage et l'apprenant. Pourtant, malgré sa position intermédiaire qui influence la façon de produire des connaissances, la technologie demeure encore généralement impensée. Ainsi, dans son récent ouvrage qui aborde la didactique des langues sous l'angle épistémologique, Narcy-Combes (2005: 172-183) évoque les "potentialités" des TIC mais pour lui la technologie semble fonctionner comme la boîte noire imaginée par les cybernéticiens pour figurer un appareil complexe " dont ils n'ont rien besoin de connaitre d'autre que ce qui y entre et ce qui en sort" (Latour, 2005 : 26).

24 La position défendue ici est que la technologie comporte une incidence profonde sur la démarche scientifique. La technologie n'est pas une donnée autonome ni indépendante. Elle devrait impliquer tout d'abord une collaboration avec des spécialistes de disciplines (ergonomie, informatique, infographie) dont la formation est éloignée de la didactique des langues et elle va obliger le chercheur en didactique à se décentrer par rapport à son objet de recherche, à l'observer à travers des points de vue différents tout en visant à les rendre solidaires pour aborder l'apprentissage des langues de manière renouvelée. D'autre part, cette position entend remettre en cause le modèle hiérarchique qui pose, trop souvent dans la littérature sur les TICE, la science comme unique déclencheur de la création et cantonne la technologie dans une position subalterne et applicative. Elle s'inscrit plutôt dans la lignée du modèle interactif qui considère que science et technologie sont toutes deux "inventives et productrices de connaissances propres" et que les transferts entre l'une et l'autre "passent par des personnes et vont dans les deux sens tout en empruntant de nombreux détours" (Vinck, 1995 : 228).

\subsection{Mode de production des connaissances}

Le schéma suivant (figure 1), adapté de Guichon (2006), permet de visualiser les différentes étapes d'un projet de recherche-développement. Il ne s'agit pas ici de les détailler toutes, mais de mettre en valeur la singularité de la recherche-développement et de déterminer les moments principaux où intervient la production de connaissances.

La recherche-développement se nourrit en principe de l'examen des travaux théoriques accessibles et de réalisations antérieures en assurant une fonction de veille scientifique et technologique qui permet de ne pas réinventer des solutions déjà existantes, mais de concevoir au contraire des applications plus abouties. La mise à jour constante des connaissances se conjugue avec la fonction prospective de la recherche-développement qui se ménage du temps pour concevoir des modèles théoriques, des applications technologiques ou des combinaisons inédites, avant de les tester selon des critères sociaux (utilité), scientifiques (validité), technologiques (robustesse et interopérabilité), ergonomiques (utilisabilité) et économiques (faisabilité et rentabilité). 
Figure 1 : Les étapes d'une recherche-développement.

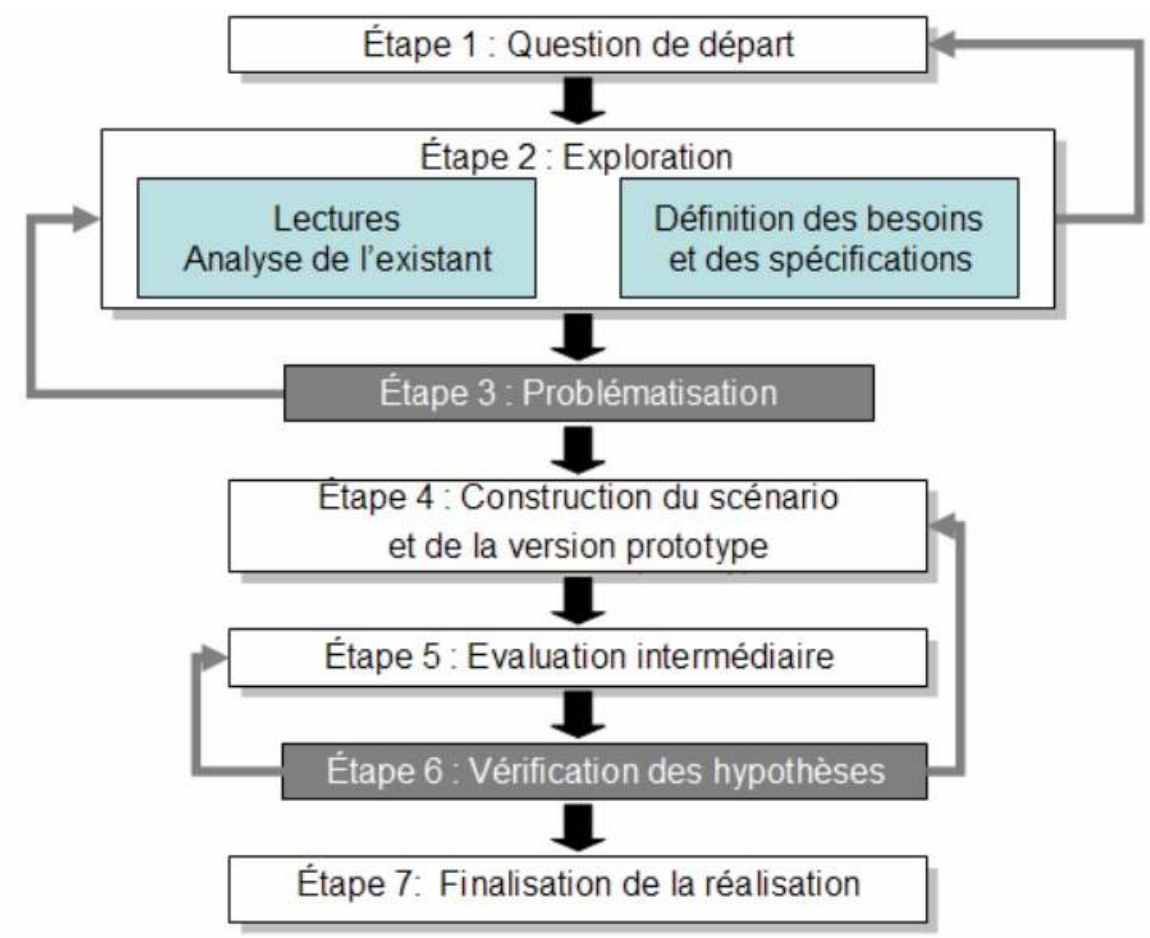

D'autre part, la recherche-développement repose sur un processus itératif (symbolisé par les flèches latérales dans le schéma) qui impose de nombreux allers-retours, d'une part, entre des équipes aux spécialités diverses et, d'autre part, entre les applications successives et des échantillons d'utilisateurs finaux. L'application traverse alors différentes phases allant du premier prototype (version beta) au délivrable (version client).

Deux moments semblent particulièrement importants en ce qui concerne la génération de connaissances nouvelles, celui de la problématisation (étape 3) et celui de la vérification des hypothèses (étape 6). La problématisation correspond à la reconfiguration du problème initial en une première ébauche de solution inédite, suffisamment aboutie pour fournir un cadre, suffisamment ouverte pour ménager des amendements ultérieurs. C'est une étape critique car elle suppose non seulement de poser toutes les données du problème, mais également de présenter des apports technologiques ou pédagogiques qui constitueront une valeur ajoutée par rapport à l'existant. Le second moment majeur de production de connaissances intervient lorsqu'une version avancée du logiciel est testée pour évaluer les apprentissages et que les différentes hypothèses qui ont présidé à sa mise au point sont validées ou invalidées.

Hoc et Darse (2002: 4) qui abordent l'activité de conception selon une perspective ergonomique établissent une distinction entre la science qui suppose de produire des connaissances et l'ingénierie pédagogique qui implique une réponse appropriée à un besoin pragmatique. La recherche-développement présente la particularité de générer des connaissances tout en tentant de mettre au point des solutions à des problèmes pédagogiques. La didactique des langues se prête bien à une association avec la technologie. Quand la technologie invente des applications pour pallier les limitations 
physiques et cognitives des hommes, la didactique des langues cherche à établir les conditions optimales pour faciliter l'apprentissage et l'enseignement des langues.

Toutefois, il est primordial de distinguer la recherche-développement et la production définitive du logiciel car cette dernière appartient à la seule catégorie des applications. En effet, les applications n'ont de valeur épistémologique qu'à la seule lumière du processus itératif entre théorisation et conception, entre recherche et développement. La théorie contribue, en effet, à extirper la recherche de la conception et à décontextualiser l'action d'un terrain particulier. Elle est "l'acide" dont parle Bachelard (1960: 52) qui travaille l'objet pour lui donner une nouvelle forme. C'est pourquoi il est souhaitable que l'objet final n'éclipse pas le sujet de la recherche, qu'il demeure une hypothèse de travail, un prétexte pour étudier le potentiel de la conjugaison de la technologie et de la didactique.

\subsection{Vers une définition de la recherche-développement contextualisée}

De la même façon que le terme recherche-développement ressortit parfois d'un abus de langage pour désigner la fabrication de ressources sur support numérique, ce terme a également besoin d'être précisé si on veut l'adopter pour décrire certaines recherches menées en didactique des langues. Les deux sections précédentes nous conduisent à proposer la notion de recherche-développement contextualisée que nous définissons ainsi: la recherche-développement contextualisée est une méthodologie de recherche didactique et technologique, qui procède par un mouvement de problématisations successives et par la mise en place d'expérimentations systématiques.

Cet aller-retour entre savoirs codifiés et prototypes débouche sur une solution suffisamment aboutie pour pouvoir tester les hypothèses initialement émises au sujet de l'apprentissage des langues et des conditions à réunir pour que celui-ci ait lieu. La qualité d'une recherche-développement contextualisée dépend de la valeur ajoutée permise par la conjugaison des connaissances en didactique et en technologie. À l'inverse de la recherche-développement industrielle, elle privilégie le processus sur le produit, la théorisation sur l'application, et elle s'inscrit dans un contexte particulier. Cette contextualisation de la recherche est la condition pour que le chercheur appréhende tous les enjeux de l'apprentissage institutionnel (sociaux, pédagogiques, techniques et économiques) avant que la solution développée puisse, éventuellement, être essaimée vers d'autres contextes que celui dans lequel elle a d'abord été mise au point.

\section{Contextualisation et décontextualisation : à la recherche d'un modèle satisfaisant}

Comme le rappellent Legros et Crinon (2002: 21), les conditions émises et les outils méthodologiques élaborés suite à une recherche-action ou à une recherchedéveloppement sont de l'ordre des "recommandations contextualisées [plutôt que] des recommandations généralisantes". Ainsi, la contextualisation de ce type de recherches constitue une indéniable limite à la portée de ses résultats (Chapelle, 2003 : 79) car elles aboutissent à des solutions technico-pédagogiques fonctionnant en local, c'est-à-dire dans un microclimat favorable à l'innovation qui s'avère difficilement reproductible ailleurs. Il est dès lors possible d'avancer que l'essaimage du produit d'une recherche- 
développement vers un autre contexte peut avoir valeur de test quant à la validité des hypothèses émises et à la qualité de la solution mise au point.

Toutefois, les universités de sciences humaines et sociales ne possèdent que rarement les moyens d'aller au-delà du prototype et d'essaimer le produit de la recherche en didactique des langues vers d'autres contextes. Deux raisons peuvent expliquer cela : elles manquent de personnes compétentes (ergonomes, informaticiens, infographistes) pour accompagner la réalisation du projet et aboutir à une solution suffisamment robuste pour être transférée. D'autre part, les investissements nécessaires pour concevoir un dispositif (logiciels, matériel, paiement d'heures de production) sont souvent insuffisants et confinent les concepteurs qui ne bénéficient pas d'un soutien institutionnel suffisant à des solutions "bricolées". Le manque de moyens humains et financiers, doublé parfois par des résistances idéologiques, condamne donc la plupart des projets à alimenter le "cimetière de prototypes" et à creuser l'écart entre les préconisations de la didactique des langues et les dispositifs médiatisés réellement proposés pour l'apprentissage des langues.

Certains produits de recherche-développement contextualisée parviennent toutefois à être essaimés vers d'autres contextes. Ainsi, le soutien financier, humain et institutionnel apporté par l'université de Lyon 2 a permis à l'équipe de conception de Virtual Cabinet (Guichon, 2004) d'aboutir à un logiciel répondant à des critères techniques et pédagogiques rendant possible son utilisation dans d'autres universités. Un accord entre trois institutions (Lyon 2, Lille 3 et Grenoble 3) a initié une collaboration visant à développer le logiciel dans d'autres langues (français langue étrangère et italien) afin de mettre les dispositifs à disposition des étudiants des institutions partenaires.

Le deuxième exemple d'un développement abouti concerne le projet Gammes d'écriture, issu d'une recherche initialement menée à l'Istituto Tecnologie Didattiche de Gênes et nourri par la thèse de Mangenot (1995). C'est en partenariat avec ce dernier que le CNDP (Centre National de Documentation Pédagogique) a reprogrammé le logiciel et le système auteur et a inclus les propositions pédagogiques pour développer un logiciel qui a été principalement utilisé dans des collèges.

Ces deux exemples, l'un reposant sur une communauté de partenaires permettant de répartir les coûts de conception, l'autre sur la collaboration d'un chercheur avec un organisme public, constituent deux possibilités pour que la recherche sur l'apprentissage médiatisé trouve des débouchés éditoriaux correspondant à des critères technicopédagogiques satisfaisants. Un troisième modèle, qui reste à construire car il ne correspond pas au fonctionnement de la recherche universitaire française, consisterait à créer des passerelles entre chercheurs et entreprises privées. Ce modèle de collaboration achoppe sur les résistances des universités de sciences humaines et sociales méfiantes vis-à-vis des impératifs financiers et juridiques auxquels sont soumis les acteurs du secteur privé. Il bute également, comme nous l'avons montré dans la première partie, sur des objectifs divergents entre chercheurs en didactique des langues et concepteurs multimédias. Pourtant, une recherche-développement dans le domaine de l'apprentissage médiatisé gagnerait à sortir d'un fonctionnement artisanal en disposant de moyens importants et en s'appuyant sur une équipe pluridisciplinaire qui combine des compétences pédagogiques, scientifiques et informatiques. Un partenariat avec des éditeurs privés pourrait apporter les moyens de développer des solutions répondant à la double contrainte de pertinence pédagogique (réponse à des besoins identifiés) et d'utilisabilité technologique. 
Quel que soit le mode de développement choisi (convention entre universités, partenariat avec des éditeurs publics ou privés), il est raisonnable d'avancer que l'effort de décontextualisation constitue le meilleur moyen pour combler les décalages constatés entre "les caractéristiques des ressources proposées sur le marché et les résultats des préconisations de la recherche" (Demaizière, 2004).

\section{Conclusion : vers une recherche impliquée}

Dans cet article, nous avons montré que recherche-développement et recherche-action partagent plusieurs caractéristiques, en particulier celle d'impliquer le chercheur en didactique des langues dans son objet d'étude. La principale distinction entre les deux méthodologies vient de ce que l'une est tendue vers l'objet (un résultat tangible, mesurable et utilisable), quand l'autre aboutit à un résultat plus diffus, davantage orienté vers le projet.

D'autre part, nous nous sommes efforcés de définir la recherche-développement comme méthode possible de production scientifique en didactique des langues et nous avons été amenés à proposer la notion de recherche-développement contextualisée qui allie une méthodologie rigoureuse, des expérimentations systématiques et la production de connaissances. Toutefois, la recherche en didactique des langues ne peut se contenter de simples préconisations. En effet, dans la lignée de Demaizière (2004) qui prône une didactique "interventionniste", la recherche-développement semble pouvoir constituer une méthodologie à même de générer des connaissances sur l'apprentissage des langues qui peuvent être investies dans des ressources ou des dispositifs médiatisés, puis étudiées auprès $d u$ public cible selon une démarche itérative et incrémentale soucieuse d'améliorer l'offre technico-pédagogique.

Enfin, nous avons montré que la conception pouvait aller au-delà du prototype et de la simple expérimentation locale si le chercheur accepte de collaborer avec des spécialistes de la technologie et d'essaimer son innovation vers un autre contexte. Cette décontextualisation, équivalente en somme à une mise sur le marché, est conditionnée par la mise en place de partenariats de divers types (avec d'autres universités, des organismes publics ou des éditeurs privés), nécessaires pour extirper le chercheur de son contexte initial et lui permettre d'aboutir à une solution présentant une valeur ajoutée pédagogique et technologique grâce aux travaux de recherche en didactique des langues.

\section{BIBLIOGRAPHIE}

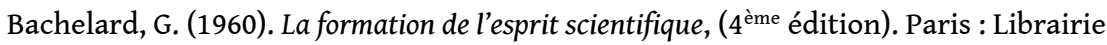
philosophique Vrin.

Catroux, M. (2002). "Introduction à la recherche-action : modalités d'une démarche théorique centrée sur la pratique". Cahiers de l' APLIUT. vol. 21, $\mathrm{N}^{\circ} 3, \mathrm{pp} .8-20$. 
Cazade, A. (2000). Recherche-développement en didactique multimédia de l'anglais. Document de synthèse pour soutenir une habilitation à diriger des recherches. Université de Poitiers.

Chapelle, C. A. (2003). English Language Learning and Technology. Amsterdam : John Benjamins. Demaizière, F. (2004). "Situer la didactique dans le processus de conception de ressources pédagogiques sur support numérique". Les cahiers de l'ACEDLE. $\mathrm{N}^{\circ} 1$.

Depover, C., Quintin, J.-J., De Lièvre, B. (2000). "La conception des environnements d'apprentissage : de la théorie à la pratique / de la pratique à la théorie". Alsic, vol. 3, $\mathrm{n}^{\circ} 1$. pp. 3-18.

Guichon, N. (2004). Compréhension de l'anglais oral et TICE : les conditions d'un apprentissage signifiant. Thèse de Doctorat. Université de Nantes.

Guichon, N. (2006). Langues et TICE. Méthodologie de conception multimédia. Paris : Ophrys. Hoc, J.-M. \& Darses, F., (dir., 2004). Psychologie ergonomique : tendances actuelles. Paris : PUF Latour, B. (2005). [1987] La science en action. Introduction à la sociologie des sciences. Paris : La découverte.

Le Diberder, A. (2003). La Recherche \& Développement dans le jeu vidéo. Conférence donnée à l'Agence Française pour le Jeu Vidéo le 19 juin 2004. http://www.afjv.com/ press0306/030617_clve.htm (consulté en décembre 2006)

Legros, D. \& Crinon, J. (2002) Psychologie des apprentissages et multimédia. Paris : Armand Colin.

Mangenot, F. (2002). «L'apprentissage des langues » in Legros D. \& Crinon J. (dirs). Psychologie des apprentissages multimédias. Paris : Armand Colin. pp. 128-153

Mangenot, F. (1995). Aides logicielles pour apprentis scripteurs en langue maternelle et en langues étrangères. Thèse de doctorat. Université de Nanterre.

Manuel De Frascati (2002). Méthode type proposée pour les enquêtes sur la recherche et le développement expérimental. OCDE.

Narcy-Combes, J.-P. (2005). Didactique des langues et TIC : vers une recherche-action responsable. Paris : Ophrys.

Pothier, M. (2003). Multimédias, dispositifs d'apprentissage et acquisition des langues. Paris : Ophrys.

Poussard, C. (2000). La compréhension de l'anglais oral et les technologies éducatives. Thèse de doctorat. Université de Paris 7.

Rézeau, J. (2001). Médiatisation et médiation pédagogique dans un environnement multimédia : Le cas de l'apprentissage de l'anglais en Histoire de l'art à l'université. Thèse de Doctorat. Université de Bordeaux 2.

Van Lier, L. (1996). Interaction in the language curriculum, awareness, autonomy \& authenticity. London : Harlow.

Vinck, D. (1995). Sociologie des sciences. Paris : Armand Colin.

\section{Sites Internet}

OMPLEP (Observatoire du Multimédia Ludo Educatif et Pédagogique) (2006). Communiqué de Presse. http://www.ludovia.org (consulté en septembre 2006). 


\section{Logiciels}

Audioster (2006). Logiciel d'apprentissage de l'anglais. Edulang http://audioster.com/ (consulté en décembre 2006)

Chanier, T., Pothier, M. \& Lotin, P. (1996). L'acte de vente. Deuxième cédérom de "CAMILLE

Travailler en France". Paris : CLE International/Nathan.

Guichon, N. \& Ghaumez, E. (2004). Virtual Cabinet. Site d'apprentissage de compréhension de l'anglais oral. http://sites.univ-lyon2.fr/vcab/ (consulté en juin 2007).

Mangenot, F. (1996). Gammes d'écriture. Logiciel Windows. Paris : CNDP.

Tell Me More Anglais (2003) Cédérom Complete beginner. Beginner. Intermediate. Intermediate +

Montigny-le-Bretonneux : Auralog.

\section{NOTES}

1. Les informations présentées sont extraites d'entretiens réalisés auprès des responsables des deux entreprises citées.

2. A noter que les projets présentés ont été menés dans le cadre national. Il serait particulièrement intéressant de connaître la situation au niveau européen et nord-américain.

3. La plupart sont des enseignants du second degré détachés dans l'Enseignement supérieur.

4. On peut également mentionner le LIUM (Laboratoire d'Informatique de l'Université du Maine)

5. Cette reconnaissance reste d'ailleurs fragile comme le fait remarquer Chapelle (2003: 77) : [I]f faculty in higher education almost by definition are considered ineligible for tenure if they work in CALL, the entire field remains the unwelcome stepchild of the larger profession of language teaching (...).

\section{RÉSUMÉS}

Cet article vise à analyser le potentiel de la recherche-développement pour la recherche en didactique des langues. En partant de son acception pour le secteur industriel dont cette démarche est issue, il s'agira de définir ce que pourrait être une recherche-développement contextualisée, de décrire son mode particulier de production des connaissances afin d'en souligner les éléments essentiels et les limites pour la recherche sur l'apprentissage médiatisé des langues étrangères.

This article aims at analysing the potential of contextualised research-development for research in applied linguistics. Starting from the meaning this methodology has for the industrial sector in which it originated, our purpose will be firstly to propose a definition for contextualised research-development, and secondly to describe its particular mode of production of knowledge so as to highlight its key elements and its limits for research on foreign language learning through multimedia. 
INDEX

Mots-clés : recherche-développement, technologie, (dé)-contextualisation

Keywords : research-development, technology, (de)-contextualisation

\section{AUTEUR}

\section{NICOLAS GUICHON}

Nicolas Guichon est maître de conférences à l'Université de Lyon 2 et chercheur au sein du laboratoire ICAR. Ses recherches portent sur l'apprentissage médiatisé et sur la

professionnalisation des enseignants utilisant les TICE. Il est le chef de projet de Virtual Cabinet, un site d'apprentissage de l'anglais.

Courriel : nguichon[at]club-internet.fr

Adresse : Université Lumière, 5 avenue Pierre Mendès-France, 69676 Bron Cedex, France. 